RAFAEL ARAGÜÉS ALIAGA

\title{
Crítica y refutación lógica del spinozismo
}

\section{Critique and logical refutation of spinozism}

RESUMEN: En la última sección de la Lógica de la Esencia Hegel lleva a cabo una profunda confrontación con la filosofía de Spinoza. Esta confrontación se articula en una crítica primero y en una refutación definitiva del spinozismo después. Ambas son esenciales para entender la metafísica hegeliana misma.

PALABRAS CLAVE: HEGEL - SPINOZISMO

- METAFÍSICA - CIENCIA DE LA LÓGICA
ABSTRACT: In the last section of the Logic of the Essence Hegel carries out a profound discussion with Spinoza's philosophy. This discussion consists first in a critique and then in the definitive refutation of Spinozism. Both issues are essential for the comprehension of Hegel's metaphysics.

KEY WORDS: HEGEL - SPINOZISM METAPHYSICS - SCIENCE OF LOGIC

$\mathrm{E}$ SCRIBE Hegel en sus Vorlesungen über die Geschichte der Philosophie que Cquien empieza a filosofar ha de ser spinozista. ${ }^{1}$ La condición personal primera para filosofar es olvidarse de las cosas particulares, alcanzar el grado de madurez intelectual que nos permite reconocer que las cosas finitas no tienen verdad en y por sí mismas, sino que toda su verdad y su sentido se encuentran en algo otro, más profundo. La tarea es, por tanto, elevarse de lo sensible al pensar. Así, el gran error que comete Jacobi, según lo expone Hegel en Creer y saber, es dar a la realidad mundana el valor de verdad absoluta, cuando es precisamente

[1] G.W.F. HegeL, Werke in zwanzig Bänden. Auf der Grundlage der 'Werke' von 1832-1845 neu edierte Ausgabe, hrsg. v. Eva Moldenhauer und Karl Markus Michel. 20 vols. Frankfurt/M: Suhrkamp 1970 (Theorie-Werkausgabe Hegels). TWA 20, 165. 
lo contrario lo que es necesario en filosofía. ${ }^{2}$ Este paso inevitable si se quiere llegar a filosofar lo representa para Hegel la filosofía spinozista, en la medida en que en ella esta asumido que todas las cosas particulares y mundanas no son más que accidentes de un otro, nulas en sí mismas. Su ser y verdad son derivados de algo más profundo.

Sin embargo, si es verdad que decimos con Hegel que al empezar a filosofar hay que ser spinozista, también podemos completar la frase, igualmente con él, diciendo que después hay que dejar de serlo. Es decir, hay que superar la posición spinozista, porque no es la posición definitiva en filosofía. En el presente texto voy a tratar de ver los argumentos que Hegel alude para, digámoslo así, abandonar a Spinoza. Y lo voy a hacer sobre todo basándome en la Ciencia de la lógica, porque ése es el lugar en la obra de Hegel donde éste plantea los argumentos de más peso para esa superación de Spinoza.

Con la visión hegeliana de la Historia de la Filosofía como el desarrollo paulatino de la verdad y la razón conecta inequívocamente la diferencia tajante que encontramos en su obra entre la "mera» crítica externa a una posición filosófica y la refutación de la misma. La crítica consiste para Hegel en señalar desde fuera de una posición filosófica concreta sus errores. La comprensión, refutación y superación de una filosofía es por el contrario una tarea que sólo puede hacerse adoptando el punto de vista de esa filosofía. Más allá de la crítica externa a una posición filosófica, existe para Hegel la necesidad de enmarcar los conceptos fundamentales de ésta dentro de la autoexposición y autoconocimiento de la razón para mostrar, por un lado, que esos conceptos forman parte del conglomerado de determinaciones del pensar y, por otro, que constituyen un momento en el desarrollo del pensar, un momento que ha de ser superado a fin de alcanzar el punto de vista verdadero por excelencia.

La máxima de Hegel es comprender cada posición filosófica no como un punto de vista absoluto y autosuficiente, ni tampoco como contrapuesta a las demás, sino como un momento en el autodespliegue de la verdad. ${ }^{3}$ La filosofía spinozista es a este respecto un caso paradigmático. Si tomamos la metafísica o lógica especulativa de Hegel como la autoexposición y autoconocimiento de la Razón en el elemento del pensar, nos encontramos con que, dentro de este sistema del pensar y sus determinaciones, los conceptos de la filosofía spinozista ocupan un lugar muy destacado, a saber, el final de la Doctrina de la Esencia. Se trata ni más ni menos que de la antesala a la Doctrina del Concepto, allí donde se abre en palabras de Hegel «el reino de la libertad» ${ }^{4}$ y se desarrollan

[2] Cf. Hegel, TWA 2, 340ss.

[3] Hegel, tWA 3, 12.

[4] Hegel, TWA 6, 240. 
los conceptos centrales de la metafísica hegeliana - el concepto de concepto y el de idea absoluta.

La discusión con Spinoza dentro de la Lógica se realiza a dos niveles en dos capítulos diferentes: Lo absoluto y La relación absoluta. Ahí se plantea respectivamente una crítica y una refutación definitiva del spinozismo. El texto introductorio Vom Begriff im allgemeinen a la Lógica del Concepto lo explica claramente: «Ya ha sido recordado anteriormente en el segundo libro de la lógica objetiva, p. 195 Observación, que la filosofía que se sitúa en el punto de vista de la sustancia y permanece en el es el sistema de Spinoza. Ahí mismo fue igualmente mostrado el error de este sistema tanto en lo que a la forma como en lo que al contenido se refiere. Otra cosa es la refutación del mismo.. ${ }^{5}$

La nota a la que se refiere Hegel es la correspondiente al capítulo Lo absoluto. Allí, señala el ahora retrospectivamente, se ha explicado la carencia del sistema de Spinoza tanto en lo que a la forma como en lo que al contenido se refiere. Se ha hecho, en definitiva, una crítica a la filosofía spinozista. Otra cosa es, nos dice Hegel, la refutación de la misma. Una verdadera refutación, como decíamos antes, no consiste solamente en una crítica. Implica, por el contrario, una confrontación profunda con la posición filosófica en cuestión, una inmersión en ella para, desde sus propios principios y su propia dinámica, superarla. La verdadera refutación del spinozismo no deriva de lo expuesto en el capítulo Lo absoluto, sino del desarrollo lógico que parte de la determinación de la sustancia y pasando por causalidad e interacción desemboca en el concepto de concepto:

La verdadera refutación ha de entrar en la virtud del contrario y situarse en la proximidad de sus fuerzas; atacarlo desde fuera de sí mismo y tener razón ahí donde él no se sitúa no hace avanzar en la cuestión. La única refutación del spinozismo sólo puede por tanto consistir en que su punto de vista sea, en primer lugar, reconocido como esencial y necesario, pero, en segundo lugar, este punto de vista sea a partir de él mismo elevado al superior. La relación de sustancialidad, considerada totalmente en y para sí misma, se convierte en su contrario, el concepto. La exposición de la sustancia contenida en el último libro, que lleva hasta el concepto, es por tanto la única y verdadera refutación del spinozismo. ${ }^{6}$

[5] «Es ist schon früher im zweiten Buch der objektiven Logik S. 195 Anm. erinnert worden, daß die Philosophie, welche sich auf den Standpunkt der Substanz stellt und darauf stehenbleibt, das System des Spinoza ist. Es ist daselbst zugleich der Mangel dieses Systems sowohl der Form als [der] Materie nach aufgezeigt worden. Ein anderes aber ist die Widerlegung desselben.» HegeL, TWA 6, 249.

[6] «Die wahrhafte Widerlegung muß in die Kraft des Gegners eingehen und sich in den Umkreis seiner Stärke stellen; ihn außerhalb seiner selbst anzugreifen und da Recht zu behalten, wo er nicht ist, fördert die Sache nicht. Die einzige Widerlegung des Spinozismus kann daher nur darin bestehen, daß sein Standpunkt zuerst als wesentlich und notwendig anerkannt werde, 
Tenemos por tanto una crítica a la filosofía de Spinoza que se deriva del capítulo Lo absoluto y una verdadera refutación del spinozismo en la exposición de la sustancia, es decir, en el desarrollo lógico que va desde la determinación de la sustancia hasta el concepto de concepto. Pasemos ahora a ver en detalle ambas, tanto la crítica como la refutación hegelianas del spinozismo.

\section{La CRÍtica de Hegel a la filosofía de Spinoza}

La exposición crítica de las ideas spinozistas la encontramos en el capítulo Lo absoluto. Se trata del primer capítulo de la última sección de la Lógica de la Esencia, La realidad efectiva (Wirklichkeit). En lo que sigue voy a reconstruir la argumentación de Hegel en ese capítulo, para después poder examinar la crítica que se deriva de su exposición.

La realidad efectiva se caracteriza de manera general por ser la unificación de las esferas del ser y de la esencia y, con ello, de todas las determinaciones contenidas en ambas. Lo absoluto es, en este marco, su unificación más inmediata: la identidad de todas las determinaciones de ser y esencia. Como tal se trata de un pensamiento que no puede ser más determinado, ya que es más bien la conjunción de todas las determinaciones, y por tanto, la anulación de toda determinación concreta. A lo absoluto no se le puede atribuir ningún ulterior predicado, y aparece en este sentido como lo totalmente vacío.

La idea de un absoluto implica por tanto la disolución de toda determinación, de tal manera que ni puede determinarse, ni es susceptible de devenir o de reflexión alguna. Sólo queda una exhibición (Auslegung) de lo absoluto mismo, un mostrar lo que es (ein Zeigen dessen, was es ist), que no consiste en más que en contemplar la identidad absoluta, como Schelling ya había afirmado en su filosofía bajo el concepto de intuición intelectual. Esta exhibición es primeramente sólo negativa: lo absoluto se muestra como el abismo de toda determinación y la identidad indeterminada de todo. Pero en la medida en que las determinaciones precedentes del ser y de la esencia han demostrado ser finitas, encuentran su fundamento en la identidad de lo absoluto. Y dado que lo absoluto es el fundamento de toda determinación y toda finitud, es posible igualmente tener en cuenta un lado positivo de su exhibición. Este lado positivo consiste, en efecto, en el conocimiento verdadero y profundo de lo finito, que como se ha mostrado en la Lógica, está conectado indefectiblemente con lo absoluto como su fundamento. Un verdadero conocimiento de lo concreto

daß aber zweitens dieser Standpunkt aus sich selbst auf den höheren gehoben werde. Das Substantialitätsverhältnis, ganz nur an und für sich selbst betrachtet, führt sich zu seinem Gegenteil, dem Begriffe, über. Die im letzten Buch enthaltene Exposition der Substanz, welche zum Begriffe überführt, ist daher die einzige und wahrhafte Widerlegung des Spinozismus.» Hegel, TWA 6, 250s. 
significa por tanto un conocimiento de lo absoluto, como Spinoza ya había señalado en su Ética: "Cuanto más conocemos las cosas singulares, tanto más conocemos a Dios. $\rangle^{7}$ Lo finito es un medio para el conocimiento de lo absoluto y la exhibición positiva consiste en presentar a lo absoluto bajo una determinación concreta. Por de pronto, este proceder puede parecer arbitrario, ya que no hay ningún criterio interno para preferir una determinación por encima de las demás y ha de decidirse por ende de manera externa. Pero si se presta atención, el hecho es que lo absoluto, siendo la identidad de todas las determinaciones, se encuentra ya él mismo claramente bajo una determinación desde el principio, a saber, la identidad. Por ello, lo absoluto es ya de hecho atributo, y la exhibición positiva, que pretende contemplar lo absoluto bajo una determinación finita o atributo, es su propio movimiento. Lo absoluto como identidad de todo ser y toda esencia deriva por sí mismo en atributo.

El atributo es lo absoluto tomado bajo una determinación. Dado que lo que constituye lo absoluto es la identidad, toda determinación que sea puesta en él está condenada a desaparecer en ella. Por ello, ya que todas las diferencias son nulas en y respecto a lo absoluto, el atributo tiene el mero estatus de una apariencia (Schein) de lo absoluto. El atributo es por ello una diferenciación formal y nula de lo absoluto. Su contenido es la totalidad; su forma sin embargo es inesencial. De ahí que pueda haber varios atributos de lo absoluto que lo muestren bajo distintas formas. En realidad, todo lo concreto ha de ser hundido en la identidad de lo absoluto, de manera que, a pesar de que Spinoza, a diferencia de Schelling, no lo reconozca, el conocimiento de las cosas singulares es en realidad su disolución. En la medida en que lo determinado es considerado como una apariencia de lo absoluto, desaparece aquello que lo hace concreto y determinado: la negación. El atributo no es por tanto solamente una apariencia de lo absoluto, sino que es puesto explícitamente como apariencia, y la exhibición positiva que utiliza al atributo como medio para acceder a lo absoluto invalida precisamente el ser del atributo mismo: el atributo es ahora, de manera explícita, simple y llanamente un modo en el que lo absoluto se expresa y emana y no tiene más consistencia propia. Se trata simplemente del modo y manera en que lo absoluto se exhibe.

Con el modo se vuelve de nuevo la identidad de lo absoluto, porque en el pensamiento del modo está incluida la idea de que lo finito no es más que una apariencia que en sí misma no es nada ni tiene consistencia alguna, sino que sólo es una manifestación de lo absoluto. En verdad, por tanto, se retorna con ello a la identidad indeterminada de lo absoluto. La exhibición de lo absoluto no consigue, en definitiva, penetrar en el interior de lo absoluto, no consigue ir más allá de su ciega identidad, pero con todo, lo absoluto ha impuesto su

[7] B. SpInoza, Ética, tr. Vidal Peña, Madrid, Alianza, 2007. Parte quinta, prop. 24. 
identidad como un manifestarse de sí mismo. El modo es la exterioridad nula de lo absoluto, su manifestarse dentro de sí mismo. De esta manera, lo absoluto se ha desvelado como un vacío manifestarse: vacío porque aquello que se muestra no es más que el mostrar mismo.

Como se ve, en el capítulo Lo absoluto nos encontramos, sin duda, con una exposición de las ideas fundamentales del spinozismo. Hegel mismo lo señala en la observación al capítulo. «El concepto de la sustancia spinozista corresponde al concepto de lo absoluto y a la relación de la reflexión para con él, tal y como se ha expuesto aquí.» ${ }^{8} \mathrm{El}$ desarrollo de los conceptos de absoluto, atributo y modo supone su asimilación dentro de la lógica especulativa, pero ilustra igualmente las razones más profundas de crítica al spinozismo. En efecto, de la exposición puramente lógica se deriva una crítica a esta filosofía en forma de observación; una crítica que, por lo demás, es en lo fundamental muy parecida a la que encontramos, por ejemplo, en las Vorlesungen über die Geschichte der Philosophie.

La tríada spinozista de sustancia - atributos - modos es, siguiendo la exposición lógica de Hegel, completa. A la sustancia, entendida como la identidad unificadora de todo lo real, le corresponde un manifestarse continuo, un emanar mediante atributos y modos. Sin embargo, y éste es el primer punto de crítica de Hegel, Spinoza no muestra la conexión argumentativa entre los tres conceptos, sino que los postula en forma de definiciones y los toma de la discusión filosófica de la época: de ahí están tomados los atributos del pensar y la extensión. Spinoza no explica por qué del concepto de sustancia se sigue el concepto de atributo, ni muestra por qué precisamente son ésos, pensamiento y extensión, los atributos de la sustancia única, y no otros. En verdad, Spinoza entiende que a la sustancia infinita le corresponden infinitos atributos. Pero al final centra su exposición en dos, pensamiento y extensión, sin probar, según Hegel, ni cómo se deriva en general el concepto de atributo del concepto de sustancia, ni cómo se deducen en particular pensamiento y extensión de ella. No se trata, por tanto, de que Hegel haga una interpretación malintencionada o tendenciosa de Spinoza, como le acusa Macherey, interpretando que la sustancia spinozista tiene sólo dos atributos, cuando el mismo Spinoza deja claro que tiene infinitos. ${ }^{9}$ Lo que Hegel critica en Spinoza es precisamente que de la infinita cantidad de atributos que podría tener la sustancia, Spinoza sólo se centre en dos, que además asume acríticamente de la discusión filosófica de la época y no deriva argumentativamente de la sustancia única. En verdad, el

[8] G.W.F. Hegel, Ciencia de la Lógica, volumen I, La Lógica objetiva. Edición y traducción de Félix Duque. Madrid: Abada 2011, p. 599.

[9] Cf. P. MAChereY, «Hegels idealistischer Spinoza», en M. Walther (Hrsg.), Spinoza und der deutsche Idealismus. Würzburg, Königshausen \& Neumann, 1992, pp. 146-162. 
atributo es según Spinoza aquello que el entendimiento capta como la esencia de la sustancia única; en la medida en que el entendimiento sólo distingue esos dos atributos, la exposición filosófica se centra en ellos. Si tenemos en cuenta, sin embargo, que en el sistema spinozista el entendimiento es un modo de la sustancia, se entiende claramente por qué Hegel ve problemático este paso de la sustancia a los atributos.

Este primer punto de crítica conecta con una crítica general al modo geométrico de Spinoza. La forma axiomática en la que éste presenta su filosofía tiene la pretensión, según Hegel, de alcanzar una verdad irrebatible como la matemática. El método de exposición matemático es, sin embargo, totalmente inadecuado para la filosofía según Hegel. Pues la exposición matemática en forma de definiciones y axiomas presupone en realidad conceptos y representaciones comunes que deberían ser en filosofía más bien objeto de crítica y examen. Así, Spinoza presupone en su filosofía conceptos como sustancia, modo, atributo, finitud e infinitud o Dios, entre otros. Para Hegel, por el contrario, la filosofía no puede hacer ni esas ni ninguna otra suposición; la filosofía ha de ser radicalmente crítica. El comienzo no puede ser más que lo más inmediato e indeterminado, el ser; o bien, desde el punto de vista de la conciencia individual, el aquí y el ahora. Un concepto como el de sustancia presupone, siguiendo la Lógica hegeliana, todo el desarrollo de la Lógica del Ser y casi toda la Lógica de la Esencia; no debería por tanto ser tomado como un punto de partida absoluto. En general, la filosofía no puede empezar por definiciones ni por axiomas, pues esto implica peticiones de principio que ella, como el pensar radical por excelencia que es, no puede permitirse.

Spinoza parte de la definición de sustancia como aquello que es en sí y se concibe por sí mismo. En pocos pasos, la sustancia es caracterizada como causa de sí misma. Sin embargo, el concepto de sustancia que opera posteriormente de verdad en el sistema de Spinoza es el de la identidad de todo lo diferente. Para Hegel, Spinoza y con él también Schelling no llegan a concebir lo absoluto o la sustancia más que como una mera identidad. Ambos caracterizan lo absoluto como causa sui, pero en ambos sólo tiene un papel relevante la comprensión de lo absoluto como identidad y unificación del todo. Así las cosas, no es posible un conocer inmanente a la sustancia. Dado que Spinoza no conoce la negación absoluta, es decir, la negación que se niega a sí misma, no entiende la sustancia como una realidad que se autodetermina, sino simple y llanamente como una identidad de todo lo diferente. Por ello el conocimiento sobre la sustancia es siempre externo. No puede partir de ella misma, ya que en su identidad vacía no hay nada que conocer, y el conocimiento se mueve así en la pura apariencia de la sustancia, entre atributos y modos.

Pasemos al tercer y último punto sobre el que bascula la crítica de Hegel. La loable intención de Spinoza es considerar todo sub specie aeterni. Sin embargo, 
el tercer grado de conocimiento, por el cual el conocimiento de lo singular lleva al conocimiento de Dios, significa en el fondo una disolución de lo singular en la identidad de la sustancia. Recuérdese la ya citada proposición 24 de Ética V: «cuanto más conocemos las cosas singulares, tanto más conocemos a Dios.» En verdad, este modo de conocer deriva del carácter de las cosas singulares como modos que expresan la esencia del todo. Spinoza no plantea un panteísmo en el que todo lo singular sea divino, como enfatiza repetidamente Hegel. ${ }^{10}$ Más bien se trata, según la lectura hegeliana de Spinoza, de comprender la nulidad de las cosas finitas, de entender que su ser es derivado, que no son en sí mismas, sino que se trata de meras modificaciones de la sustancia única y absoluta mediante las cuales esta emana y se manifiesta. El conocer que tiene esto presente y considera lo finito sub specie aeterni es la ciencia intuitiva. La ciencia intuitiva empieza desde el atributo, la esencia de la sustancia, y baja a lo singular. Ella implica, de esta manera, la reducción de lo finito a la sustancia única. El desarrollo lógico del concepto de absoluto en Hegel muestra sin embargo que este modo de conocer implica en realidad una disolución de lo finito en el magma de la identidad originaria. El conocimiento del todo reduce lo concreto a nada. Lo concreto desaparece aquí en el abismo de lo general, en la identidad del todo.

Spinoza requiere del pensar la sublime exigencia de considerarlo todo bajo la figura de la eternidad, sub specie aeterni, o sea, tal como ello es dentro de lo absoluto. Pero, dentro de aquel absoluto que es solamente la identidad inmóvil, tanto el atributo como el modo están solamente como evanescentes, no como cosas que devienen, de suerte que, con ello, ese desaparecer toma igualmente su inicio positivo solamente de fuera. ${ }^{11}$

Como vemos, la filosofía que propone Spinoza y que plantea la sustancia única como la identidad de todo lo real es incapaz por ello, en primer lugar, de derivar de su principio lo finito. Ni los atributos ni la multiplicidad de los modos pueden entenderse en su especifidad desde la identidad de la sustancia, de tal manera que han de ser distinguidos desde fuera de la sustancia, por el entendimiento, y tomados de manera acrítica. Pero además, en segundo lugar, esta filosofía no puede más que, de acuerdo con su mismo principio, reducir atributos y modos a la identidad de la sustancia, hacerlos desaparecer en el todo de la identidad única. Se añade a esto, en tercer lugar, un modo de proceder geométrico inadecuado para la filosofía. Esta es, en lo fundamental, la crítica de Hegel a Spinoza. Pasemos a ver ahora lo que Hegel llama la refutación del spinozismo.

[10] Cf. las notas a Enc. $\$ \$ 50$ y 573 .

[11] Hegel, CL, 601. 


\section{LA REFUTACIÓN DEL SPINOZISMO}

Hasta este punto hemos examinado la crítica hegeliana a la filosofía de Spinoza. El desarrollo lógico expuesto en el capítulo Lo absoluto se corresponde con la filosofía de Spinoza, veíamos, porque en su sistema la sustancia cumple el rol de identidad absoluta e indeterminable de todo lo real. En la medida en que la crítica de Hegel se basa en el propio desarrollo de este pensamiento de la unidad idéntica de toda determinación en un absoluto, y en la medida, igualmente, en que este absoluto entendido como identidad es superado, parece claro, no obstante, que no estamos simplemente ante una crítica externa a la filosofía de Spinoza. Siguiendo el criterio que Hegel mismo pone para hablar de refutación, y no solamente de crítica, hay que reconocer que la crítica a Spinoza derivada del capítulo Lo absoluto podría verse sin lugar a dudas como una refutación de pleno derecho.

¿Por qué entonces dice Hegel que la refutación del spinozismo es otra cosa? La razón esta en que el spinozismo, como posición filosófica, no se identifica sencillamente con la filosofía que Spinoza expone en su Ética. Si bien Spinoza plantea correctamente los términos del spinozismo, a juicio de Hegel el desarrollo que de hecho él realiza no es el adecuado. Pues Spinoza no toma ni desarrolla el spinozismo desde su raíz, a saber, desde el concepto de sustancia como causa de sí misma. Ni la filosofía de Spinoza es spinozismo en su forma más acabada, ni su refutación es por ende una refutación del mismo.

Con todo, cabe igualmente preguntarse por qué Hegel cree necesaria una refutación del spinozismo. La respuesta es que dentro de la filosofía idealista de la época el debate sobre la irrefutabilidad del spinozismo adquirió enorme relevancia. Schelling había abierto el debate afirmando en sus Cartas sobre dogmatismo y criticismo que de la filosofía kantiana se derivaba la oposición de dos filosofías contradictorias entre sí: el dogmatismo, que en su versión más acabada sería spinozismo, por un lado, y el criticismo por otro. Ambas filosofías estarían según él igualmente bien fundamentadas, lo que significa que no habría más razón para decantarse por el criticismo kantiano o el spinozismo que el gusto personal de cada cual. Hegel se hace cargo en la Ciencia de la lógica indudablemente de esta problemática. Su convicción es haber mostrado, por fin, la refutación definitiva del spinozismo, dejando claro con ello que en el dilema planteado por Schelling él se decanta por el criticismo de Kant, o más bien, por una filosofía nueva que surja a partir de él.

La sustancia única como principio idéntico del todo es una determinación del pensar y encuentra como tal su desarrollo en una lógica especulativa. En la medida en que en la época de Hegel ese pensamiento se entiende bajo el término de lo absoluto y su principal defensor, Schelling, había ya planteado lo absoluto como la identidad de todo lo diferente y el principio ontológico de todo lo real, 
se entiende que Hegel hable entonces de absoluto y no de sustancia en el capítulo en cuestión. El pensamiento de fondo es el mismo que han defendido Schelling y Spinoza con sus filosofías. Lo absoluto es la unidad de ser y esencia, y como tal ha de ser primeramente tratado en forma de unidad inmediata: identidad. Esta unidad es posteriormente enriquecida y desarrollada en la Lógica de Hegel y culmina en un concepto de absoluto que ya no es sencillamente identidad de ser y esencia, sino sustancia. La sustancia unifica ser y esencia bajo la determinación de causa de sí misma. Su esencia es su existencia así como su existencia es su esencia, en la medida en que ambas son la reflexion de la otra. De esta manera, el desarrollo de la dialéctica de la sustancia es el desarrollo de la idea de lo absoluto en su radicalidad. Cuando este desarrollo muestra, como de hecho lo hace la Lógica hegeliana, que lo absoluto no es la verdad última, sino que es una determinación más del pensar que ha de dar paso a un punto de vista más elevado, nos encontramos con la verdadera refutación del spinozismo.

¿Cuáles son las claves de esta refutación del spinozismo? En el núcleo del pensamiento de sustancia se encuentra la necesidad absoluta. La necesidad absoluta es la última de las determinaciones de la realidad efectiva. Consiste en el ser que es, porque es: la mediación del ser consigo mismo. Lo absolutamente necesario lo es sin referencia a ningún otro, sino en y por sí mismo necesario. Con ello, la necesidad absoluta unifica ser y esencia, pero esta vez no como identidad indeterminada, sino como la unidad evolucionada de la sustancia. La sustancia es por sí misma, es, como afirma Spinoza, en sí y se concibe por sí misma. En este punto se alcanza el verdadero despliegue de lo absoluto:

El absoluto, exhibido por de pronto por la reflexión externa, se exhibe ahora a sí mismo como forma absoluta o como necesidad; esta exhibición de sí mismo es su acto de ponerse-a-sí-mismo, y él es solamente este acto de ponerse. ${ }^{12}$

El ponerse-a-sí-mismo es lo fundamental de la sustancia. En ella cristaliza la unidad de ser y esencia de manera dinámica. Se trata de un ser que es reflexión de sí mismo, que se pone y se presupone. En este su autodespliegue lo absoluto no tiene ya atributos, sino accidentes: la totalidad de lo real que lo absoluto en su dinámica pone a partir de sí mismo. Sin embargo, la relación entre sustancia y accidentes deriva en la relación de causalidad. Para entender este paso hay que tener en cuenta que no se trata simplemente de que la sustancia tenga accidentes. Lo característico de la sustancia es más bien que pone sus accidentes, que ella es un poder absoluto sobre ellos.

La sustancia cumple su determinación en la medida en que se pone a sí misma. Pero su ponerse en una multitud de accidentes no es completo. La re-

[12] Hegel, CL, 619. 
flexión de la sustancia sólo puede ser en verdad otra sustancia. Lo característico de la sustancia, a diferencia de la posición kantiana, no es la permanencia en el tiempo sino la dinámica del ponerse a sí misma. En la medida en que la sustancia es esencialmente el movimiento de autoposición, su unidad se desgarra. Desplegada, la sustancia es poniente y puesta, activa y pasiva. El pensamiento de la sustancia deriva por ello en duplicidad. La sustancia única se desdobla indefectiblemente y deviene relación causal. Es en la causalidad donde la sustancia encuentra su culminación. Ahora entendida como causa, la sustancia llega a manifestarse y ponerse absolutamente en su efecto. Lo que en la accidentalidad era un movimiento fracasado, llega a cumplirse en otra sustancia, su efecto.

La relación causal, por su parte, se completa según la Lógica con el concepto de interacción, donde cabría esperar la reunificación de la sustancia consigo misma. Sin embargo, éste tampoco es el caso. O más bien, de la interacción surge una nueva unidad que ya no es la unidad de la sustancia, sino la del concepto. Nuevamente a diferencia de Kant, Hegel no piensa la interacción como una comunidad de sustancias. Pues lo característico de la interacción es que en ella ya no queda substrato alguno. En la interacción la causa es a la vez efecto, el efecto es a la vez causa. Pero causa y efecto, efecto y causa, no son ya nada más ni nada menos que eso.

Pues ella [la interacción, R. A.] contiene, en primer lugar, el desaparecer de aquel originario persistir de la sustancialidad inmediata; en segundo lugar, contiene el surgimiento de la causa y, con ello, la originariedad, en cuanto que por su negación se media consigo. ${ }^{13}$

La interacción presenta un resultado doble de todo el proceso que parte desde la sustancia. Por un lado, en la medida en que aquél persistir de la sustancialidad desaparece, se diluye la consistencia de la sustancia. Ya no queda en ningún extremo de la interacción algo que pueda ser reconocido como sustancia, sino los puros polos de la relación causal recíproca. La interacción es de este modo la pura y vacía relación causal misma, "un vacío modo y manera ${ }^{14}$ donde la causa se define por el efecto y el efecto por la causa. En su culminación en la interacción, causa y efecto no inhieren en ningún sitio; no hay ya sustancia alguna. Por otro lado, con la interacción surge un nuevo modelo de originariedad. Esta originariedad ya no es la misma que la de la causa, sino que inaugura un nuevo plano. La diferencia está en que se trata de una originariedad que resulta de la negación de sí misma.

[13] Hegel, CL, 636.

[14] Ibid. 
En la interacción tiene lugar el despliegue completo del concepto de sustancia. La sustancia implica, por un lado, unidad de ser y esencia y unidad consigo misma; por otro lado, sin embargo, unidad dinámica, diferenciante, y por ello duplicidad. La contradicción de la que está preñado este pensamiento sale a la luz completamente en la interacción. La relación sustancial y, por ende, la relación causal postulan la unidad de elementos sustancialmente diferentes, la contradicción absoluta:

Allí han desaparecido por tanto necesidad y causalidad; ellas contienen ambas cosas, la identidad inmediata como cohesión y como referencia, y la sustancialidad absoluta de los [extremos] diferenciados, y con ello, la absoluta contingencia de los mismos, la unidad originaria de la diversidad sustancial; por tanto, la contradicción absoluta. ${ }^{15}$

En estas frases de Hegel se encuentra la clave de la refutación del spinozismo. El spinozismo postula lo absoluto como la unidad originaria de la diversidad sustancial y cae así en una contradicción absoluta. Esta identidad de lo diferente sólo es posible si se abandona el pensamiento de la sustancialidad, si se transita del pensamiento de lo absoluto como unidad ontológica de la diversidad, al concepto de concepto, la unidad ideal de lo diferente. Por un lado, la apariencia del ser-otro ha caído, por otro lado, la identidad interna de la necesidad como una relación reflexiva por medio del otro ha salido a la luz. En la medida en que la sustancialidad de la necesidad ha sido dejada de lado y ha cristalizado la idea de una auto-mediación y auto-determinación, surge la estructura fundamental del concepto. Con ello se ha alcanzado a la vez el núcleo esencial de la libertad. Por eso se entra con la Doctrina del Concepto, en palabras de Hegel, en el reino de la libertad. La sustancia, liberada de las relaciones de necesidad y del azar, es el concepto, de manera que se traza una frontera clara entre sustancia y necesidad, por un lado, y concepto y libertad, por el otro.

\section{LA RELEVANCIA DE LA REFUTACIÓN DEL SPINOZISMO EN LA METAFÍSICA HEGELIANA}

La importancia de la refutación del spinozismo no se limita a una disputa entre Hegel y Spinoza. Se trata, más bien, de un elemento clave en la comprensión de la metafísica hegeliana. Como hemos visto, el spinozismo no se identifica totalmente con la filosofía presentada por Spinoza. Se trata más bien para Hegel de una posición metafísica general.

[15] Hegel, CL, 638. 
Lo absoluto es la unidad de ser y esencia. En su determinación más inmediata, esta unidad es concebida como identidad. Pero en su determinación completamente desarrollada, lo absoluto es sustancia. La metafísica de lo absoluto plantea la unidad originaria de todo lo diferente en lo absoluto. Lo absoluto es causa sui, puro autodespliegue, y en ese su autodesplegarse sienta la multiplicidad infinita de lo concreto y la reunifica como emanación suya que es. La refutación del spinozismo que Hegel presenta es la refutación a esta posición metafísica, la refutación a la filosofía de lo absoluto. La refutación del spinozismo se muestra así como un punto esencial para entender la metafísica hegeliana. No se trata de que Hegel deseche las filosofías de Spinoza o de Schelling porque en ellas no se llega al verdadero concepto de lo absoluto. Lo fundamental no es que Hegel presente un concepto de absoluto dinámico, un absoluto que sea a la vez sustancia y sujeto. Lo que Hegel plantea es, más bien, la necesidad de abandonar esa posición metafísica como tal, lo limitado de una filosofía que trate de investigar la unidad ontológica de lo real. Entender el paso de la sustancia al concepto es comprender la raíz del idealismo: que toda verdad y toda objetividad surgen del pensar. Hegel plantea por ello un cambio de paradigma, una metafísica sin ontología. No se trata de investigar el principio ontológico de todo lo real, el ser en todo ser. La tarea es más bien elaborar un sistema de la razón basado en una metafísica concebida como lógica especulativa, como ciencia del pensar y sus determinaciones. La verdad del pensar es el concepto, y es del concepto, de su dinámica y de su objetividad, de lo único que trata la filosofía.

\section{REFERENCIAS BIBLIOGRÁFICAS}

G.W.F. Hegel, Ciencia de la Lógica. I. La Lógica objetiva 1. El ser (1812), 2. La doctrina de la esencia (1813). Edición y traducción de Félix Duque. Madrid: Abada 2011. (CL)

G.W.F. Hegel, Enciclopedia de las ciencias filosóficas. Traducción de Ramón Valls Plana. Madrid: Alianza 1997. (Enc.)

G.W.F. HegeL, Werke in zwanzig Bänden. Auf der Grundlage der 'Werke' von 1832-1845 neu edierte Ausgabe, hrsg. v. Eva Moldenhauer und Karl Markus Michel. 20 vols. Frankfurt/M: Suhrkamp 1970 (Theorie-Werkausgabe Hegels). (TWA)

P. Macherey, «Hegels idealistischer Spinoza», en M. Walther (Hrsg.), Spinoza und der deutsche Idealismus. Würzburg, Königshausen \& Neumann, 1992, pp. 146-162.

B. SpInoza, Ética, tr. Vidal Peña, Madrid, Alianza, 2007. 
Rafael AragüÉs Aliaga es doctorando en la Ruprecht-Karls-Universität Heidelberg. Director: Prof. Dr. Hans Friedrich Fulda

Línea de investigación: Metafísica y Filosofía política, Hegel, Kant, Schelling, Fichte, Schopenhauer, Spinoza, Marx.

DIRECCIÓN

Eulerstraße 11a, 13357 Berlin

(+49) 15779859390

rafael.aragues@gmail.com

Publicaciones ReCientes:

«Apuntes sobre la teoría del valor de Marx», en Cuaderno de materiales, $\mathrm{n}^{\circ} 25,2013$.

«¿Es la filosofía hegeliana un acosmismo? Hegel y el concepto de mundo», en Revista Tales, $\mathrm{n}^{\circ}$ 4, 2011. 